\title{
A Simulation Engine for the Characterization of Capacity Degradation Processes in Lithium-ion Batteries Undergoing Heterogeneous Operating Conditions
}

\author{
Aramis Perez ${ }^{1,2}$, Heraldo Rozas ${ }^{3}$, Francisco Jaramillo ${ }^{4}$, Vanessa Quintero ${ }^{5}$ and Marcos Orchard ${ }^{6}$ \\ ${ }^{1}$ School of Electrical Engineering, University of Costa Rica, San Jose, Costa Rica \\ aramis.perez@ucr.ac.cr \\ 2,3,4,6 Department of Electrical Engineering, University of Chile, Av. Tupper 2007, Santiago, Chile \\ aramis.perez@ing.uchile.cl \\ heraldo.rozas@ing.uchile.cl \\ francisco.jaramillo@ing.uchile.cl \\ morchard@ing.uchile.cl \\ ${ }^{5}$ Faculty of Electrical Engineering, Universidad Tecnológica de Panamá,C.R. Panamá Oeste, Panamá \\ vanessa.quinterol@utp.ac.pa
}

\begin{abstract}
Characterizing the degradation process of lithium-ion (Liion) batteries is still a matter of ongoing research due to the diverse operating conditions at which they are submitted. For example, different current discharge rates and asymmetrical charge/discharge cycles are critical operating conditions that affect both the performance and the lifespan. This article extends and improves a previously published methodology to estimate the degradation process of Li-ion batteries with the novelty of using the Kalman Filter to estimate one of the parameters of the proposed state-space model. Furthermore, the Kalman Filter is then combined with a Similarity-BasedModeling framework, which integrates information of the State of Charge and different discharge currents in each operating cycle to estimate the degradation process. The results are obtained using information provided by the manufacturer and also with measured data. Finally, the proposed methodology is applied to a random usage profile of an ElectricVehicle to characterize the degradation process of the batteries under more realistic usage conditions.
\end{abstract}

\section{INTRODUCTION}

Typically the information related to the degradation process found on Li-ion battery datasheets explains this phenomena just under specific conditions. In a more realistic approach,

\footnotetext{
Aramis Perez et al. This is an open-access article distributed under the terms of the Creative Commons Attribution 3.0 United States License, which permits unrestricted use, distribution, and reproduction in any medium, provided the original author and source are credited.
}

a single battery can be used under different conditions during its lifetime. For instance, the initial and final values of the State of Charge (SOC), the discharge current rate, or temperature, are some of the operating conditions that affect the performance in the short and in the long term. For this reason, understanding that the degradation process of $\mathrm{Li}$-ion batteries depends on how the battery was used, becomes relevant. Since the possible combinations of operating conditions can be infinite, proposing a model that uses the available information to extrapolate the degradation process when different conditions are present becomes of utmost importance.

In a previous work (Perez, Quintero, et al., 2018), the authors proposed a Li-ion battery degradation model, which incorporates the impact of arbitrary discharge currents. This model was represented by a state-space structure, and fitted using data from a Sony battery (Sony US18650 1.4 Ah Li-ion battery (Ning \& Popov, 2004)). Even though the proposed model was initially fitted for a specific data set, it was subsequently extended to other brands of Li-ion batteries. The original results showed that the state-space model was able to characterize the degradation process properly when the discharge current was equal to its nominal value (1C). However, when the discharge current was doubled (2C), the proposed model was not able to follow the experimental degradation results, although it did have a biased trend. One of the findings demonstrated that if one of the coefficients of the previously proposed model was fitted freely, the bias was eliminated.

To improve the previous results, this article proposes the use of the same structure of the original model combined with 
a Kalman Filter (KF) based approach to resolve the bias to characterize the degradation of Li-ion batteries. This way, the model described in this article can be extended to other Li-ion batteries where only the information provided by the datasheet regarding the discharge behavior at nominal current is available. Moreover, the obtained proposed model in addition to a Similarity-Based-Modeling (SBM) framework is used to characterize the degradation process when the Li-ion battery is operated at different values of SOC, instead of the typical fully charged to fully discharged operating conditions. This combined approach is then tested on an Electric-Vehicle (EV) usage profile, where multiple SOC and C-Rate states can be present. This allows to understand in a better manner the possible degradation trends of Li-ion batteries when multiple operating conditions can be present.

\section{TheORETICAL FRAMEWORK}

The battery lifetime is affected by the degradation process suffered by the battery. Currently, several techniques are proposed to estimate this process like fuzzy logic (Salkind, Fennie, Singh, Atwater, \& Reisner, 1999), neural networks (Charkhgard \& Farrokhi, 2010), KF (Bishop, Welch, et al., 2001), Extended Kalman Filter (Bishop et al., 2001), and particle filter (Olivares, Munoz, Orchard, \& Silva, 2013). These techniques require a battery model that characterizes battery behavior. Different models are proposed in the literature according to the type of variables considered, as explained in the next subsection.

\subsection{Degradation in Li-ion Batteries}

The State of Health ( $\mathrm{SOH})$ is a concept associated with the percentage of degradation suffered by the battery in each cycle of operation. The study of the $\mathrm{SOH}$ is related to the longterm battery performance, hence the End-of-Life (EoL) of the battery can be estimated through the $\mathrm{SOH}$. This indicator allows the decision making process of how to handle and use the Li-ion battery. Furthremore, the degradation process is caused by diverse factors such as temperature, cycling rate, handling and storage, among others.

Various models have been proposed to characterize the degradation process suffered by the battery. Depending on the model characteristics, they can be classified into mathematical, electrochemical, and electrical-circuit-based models. Mathematical models use a set of equations to describe battery properties. The electrochemical models use equations that describe the chemical behavior of the battery. Furthermore, electric-circuit-based models are those that use equations obtained from equivalent electrical circuits to describe the behavior of the battery (Fotouhi, Auger, Propp, Longo, $\&$ Wild, 2016). Some authors include a separate category of empirical models when classifying the models that describe the behavior of the battery, which is a type of mathemati- cal model. Empirical models are more flexible and generally much simpler when compared to other models (Pola et al., 2015).

The models proposed consider different aspects to estimate battery degradation. For example, some models are based on measurable parameters of the battery such as changes in internal impedance, temperature, and discharge currents. This situation is exemplified in the model proposed in (Guha \& Patra, 2018) where the authors fuse two concepts: the loss of capacity that in this case is defined based on the current drained to the battery and the increase in internal impedance. Another example is presented in (Pérez et al., 2017), where the author uses the concept of Coulomb efficiency to determine the degradation in the battery. Coulomb efficiency is a concept that groups various effects that cause degradation in the battery, simplifying its estimation.

Several degradation models combine the information regarding the discharge current rates curves to characterize its behavior. For example, in (Yang, Wang, Xing, \& Tsui, 2017) the authors present a model that characterizes the capacity degradation using two terms based on a natural logarithm function. Another example is the empirical model presented in (Perez, Quintero, et al., 2018) where the model describes the process of battery degradation considering different discharge C-rates. The model consists of a discrete state-space model that takes as references the discharge curves. Other types of model describe the battery degradation considering the charging process, such as the case presented in (Ning \& Popov, 2004).

\subsection{Kalman Filter}

The KF (Kalman, 1960) is an algorithm used for estimating unknown (or not directly measurable) states by fusing current noisy measurements with the most recent estimated state (Vachtsevanos, Lewis, Roemer, Hess, \& Wu, 2007). The KF assumes that the probability density function (PDF) of the estimated state is Gaussian and, hence, it can be parameterized by a mean and a covariance (Arulampalam, Maskell, Gordon, \& Clapp, 2002). For linear systems with Gaussian noises, the $\mathrm{KF}$ is optimal in terms of minimizing the estimation-error covariance, which is obtained by the optimal computation of the so-called Kalman gain.

The linear discrete-time system can be described by a recursive difference equation with the state vector $x \in \Re^{n}$ and the sensor measurements vector $y \in \Re^{m}$ (Eq. 1)

$$
\begin{aligned}
& x(k)=A(k) x(k-1)+B u(k-1)+w(k-1) \\
& y(k)=C(k) x(k)+v(k)
\end{aligned}
$$

where $A(k)$ is the the status transition matrix, and $C(k)$ is the observation matrix. $u(k)$ and $B(k)$ are the control input 
and the input control matrix. $w(k)$ and $v(k)$ are the process noise the measurement noise, which are assumed as Gaussian distributions with covariance matrices $R_{w w}$ and $R_{e e}$, respectively.

Defining $\hat{x}(k)$ as the state estimation, $\hat{P}(k)$ the covariance for the state estimation error, $\hat{y}(k)$ the measurement estimation, and $y(k)$ an on-line measurement. The KF can be computed through a two step prediction-update algorithm (see Algorithm 1 (Candy, 2016)). On the one hand, the prediction step computes a prediction of the current estimated state ahead in time. On the other hand, the update step updates/corrects this predicted state using an on-line measurement of the observed process.

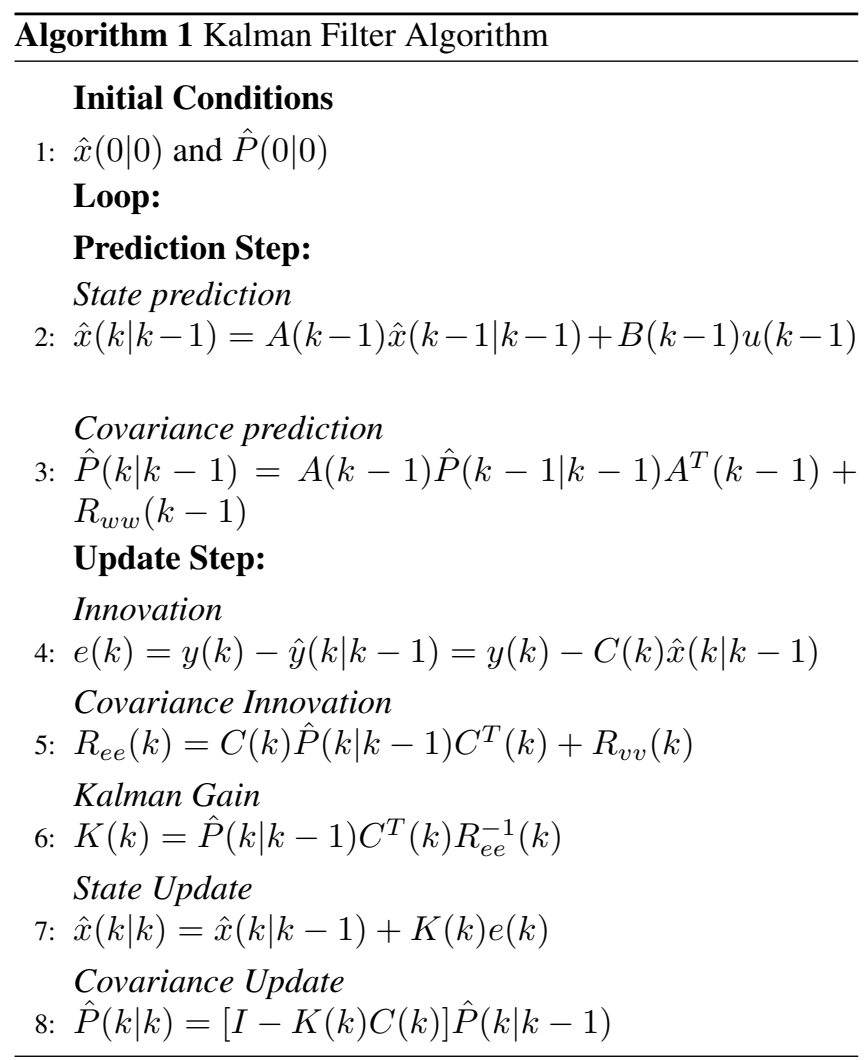

\subsection{Similarity Based Modeling}

SBM is a non-parametric technique that estimates the output of a system through historical data and compares it with the actual and measured output (Perez, Jaramillo, Quintero, \& Orchard, 2018). It is frequently used in the detection of faults of various systems (Marins, Ribeiro, Netto, \& da Silva, 2018). A system is described using Eq. 2, where $x$ is the input of the system, $y$ is the output, and $f(\cdot)$ represents an unknown function.

$$
y=f(x), \quad x \in \mathbb{R}^{m}, \quad y \in \mathbb{R}^{p}
$$

The input and output values, obtained from the database, are separated in two different matrices. Equation (3a) represents all the known inputs and Eq. (3b) is the corresponding output.

$$
\begin{aligned}
& D_{i}=\left[\begin{array}{llll}
x_{1} & x_{2} & \ldots & x_{n}
\end{array}\right] \in \mathbb{R}^{m \times n} \\
& D_{o}=\left[\begin{array}{llll}
y_{1} & y_{2} & \ldots & y_{n}
\end{array}\right] \in \mathbb{R}^{p \times n}
\end{aligned}
$$

According to (Perez, Jaramillo, et al., 2018), in SBM, for any given set of inputs $x^{*}$, the output $y^{*}$ can be estimated through a linear combination of matrix $D_{o}$ and a weighing vector denoted $w$. In other words, the estimated output $\hat{y}^{*}$ is equal to the product between $D_{o}$ and $w$, hence $\hat{y}^{*}=D_{o} w$. Equations (4) and (5) show how to calculate $w$. The similarity operator is defined as $\Delta$.

$$
\begin{gathered}
\hat{w}=\left(D_{i}^{T} \Delta D_{i}\right)^{-1}\left(D_{i}^{T} \Delta x^{*}\right) . \\
w=\frac{\hat{w}}{\mathbf{1}^{T} \cdot \hat{w}} .
\end{gathered}
$$

\section{Characterization of the Degradation Pro- CESS OF LI-ION BATTERIES}

According to (Perez, Quintero, et al., 2018), the SOH degradation process of a certain battery can be described in statespace model as follows:

\section{Transition equation:}

$$
\begin{aligned}
& x_{1}(k+1)=e^{b} x_{1}(k) \\
& x_{2}(k+1)=e^{d} x_{2}(k)
\end{aligned}
$$

\section{Observation equation:}

$$
y(k)=a x_{1}(k)+c x_{2}(k)
$$

where, $a, b, c$ and $d$ are parameters which are estimated using empirical data (for a more detailed description/analysis of each parameter, please see (Perez, Quintero, et al., 2018)). Note that this space state model is discretized in terms of the cycle number; in other words, when we say $x_{1}(k)$ that means the value of variable $x_{1}$ at cycle $k$.

As we review in section 2.1 , the battery degradation process depends on several factor, such as operation temperature, depth of discharge, current of discharge, among others. Therefore, the parameters $a, b, c$, and $d$ of the presented degradation model will vary depending on the operation conditions. In particular, in (Perez, Quintero, et al., 2018), this degradation model is studied considering the discharge current dependency. The authors estimated these parameters using the information of three degradation processes $(1 \mathrm{C}, 2 \mathrm{C}$, 
Table 1. Mean value and confidence bounds of the model coefficients.

\begin{tabular}{c|c|c|c|c}
\hline \hline Coefficient & Parameter & $1-\mathrm{C}$ & $2-\mathrm{C}$ & $3-\mathrm{C}$ \\
\hline \hline$a$ & Mean Value & 0.06108 & 0.07653 & 0.06763 \\
& Confidence bounds & $(0.06084,0.06132)$ & $(0.07371,0.07965)$ & $(0.06588,0.06937)$ \\
\hline$b$ & Mean Value & -0.02905 & -0.02896 & -0.02093 \\
& Confidence bounds & $(-0.02931,-0.02879)$ & $(-0.03165,-0.02627)$ & $(-0.02203,-0.01984)$ \\
\hline$c$ & Mean Value & 0.946 & 0.932 & 0.9376 \\
& Confidence bounds & $(0.9457,0.9462)$ & $(0.9292,0.9349)$ give & $(0.9357,0.9395)$ \\
\hline$d$ & Mean Value & -0.0001406 & -0.0002115 & -0.0003943 \\
& Confidence bounds & $(-0.0001416,-0.0001395)$ & $(-0.000225,-0.000198)$ & $(-0.0004026,-0.000386)$ \\
\hline
\end{tabular}

and 3C), and curve fitting tools. The resultant parameters are depicted in Table 1. Afterwards, they evaluated the proposed model with the estimated parameters for other degradation data sets with the aim of assessing its capability of generalization. Their results showed that using this degradation model with the parameters of Table 1 it is possible to characterize other degradation processes that are cycled at $1 \mathrm{C}, 2 \mathrm{C}$ or $3 \mathrm{C}$. Nevertheless, to accomplish more accurate characterizations, it is necessary to estimate the parameters $c$ for each particular degradation process. Otherwise, the degradation process is estimated with a bias, this fact will be illustrated in the following paragraph.

The procedure presented in (Perez, Quintero, et al., 2018) is applied in order to obtain the degradation model of a battery whose discharge current is $2 \mathrm{C}$. The results is shown in Figure 1. Despite the fact, that the fitted curve has the same trend of the experimental data, there exists an evident bias that can be fixed by changing the value of the parameter $c$ of Eq. (8) (Perez, Quintero, et al., 2018). Therefore, to compute a better estimation, it is mandatory to estimate the parameter $c$ for the particular degradation process. In the following section, we will present a sequential procedure to learn the best value for c.

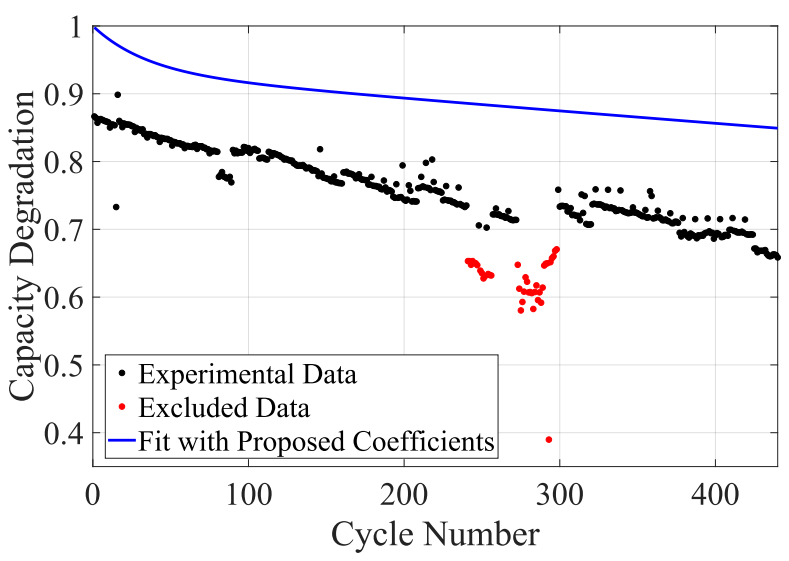

Figure 1. Fitted curve by using the model proposed in (Perez, Quintero, et al., 2018).

\subsection{Estimating the Parameter $c$ for a Degradation Pro- cess with a Fixed C-Rate}

In this case, it is assumed that the battery is cycled with a fixed C-Rate (although this information can be found in available datasheets); consequently, we have to estimate $c$ for a sole degradation process.

Firstly, note that the degradation process described in Eq. (6), (7) and (8) can be rewritten as:

$$
y(k)=a e^{b k} x_{1}(0)+c e^{d k} x_{2}(0)
$$

Then, we can introduce a modified observation equation:

$$
\begin{aligned}
\tilde{y}(k) & =y(k)-a e^{b k} x_{1}(0) \\
& =c e^{d k} x_{2}(0)
\end{aligned}
$$

Defining $\alpha(k)=e^{d k} x_{2}(0)$, it is possible to propose the following space state model:

\section{Transition equation:}

$$
c(k+1)=c(k)+\omega
$$

Observation equation:

$$
\tilde{y}(k)=\alpha(k) c(k)+\epsilon
$$

where $c(k)$ is the variable of interest and $\tilde{y}$ can be measured. While $\omega \sim N\left(0, \sigma_{\omega}^{2}\right)$ and $\epsilon \sim N\left(0, \sigma_{\epsilon}^{2}\right)$ are the process noise and the observation noise, respectively. These noises are added in the model to formulate a linear Gaussian system, which allows the application of KF to sequentially estimate the parameters $c$. KF is employed because -given this estimation framework- it offers an estimation with minimum expected square error. The estimation algorithm is summarized in Algorithm 2. It works as follows. Firstly, the parameters $a, b, c_{0}, d$ are initialized with the mean values of Table 1 (Line 1). Afterwards, it is assumed a normal distribution for $c(0)$ (Line 2). Then, the loop starts (Line3), where for each 
new cycle $\tilde{y}(k)$ is computed and KF is calculate the estimated state $c(k)$ ( Line 4 and 5).

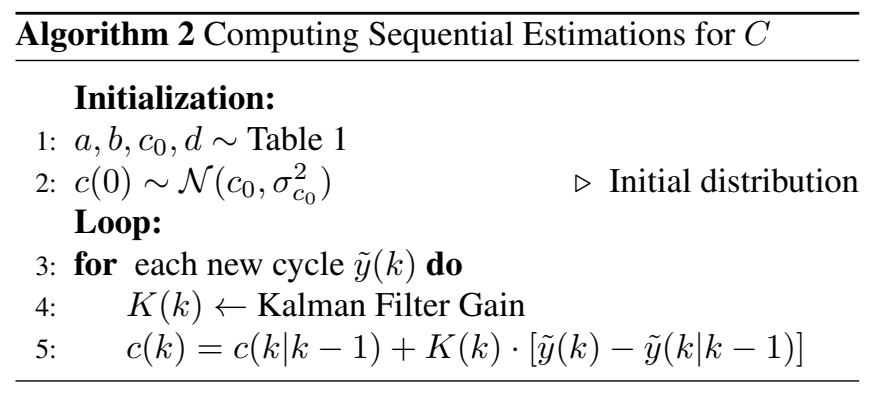

\subsubsection{Example Estimation with Fixed Discharge Current}

The sequential estimation is applied for the experimental data of Figure 1. The results for different cycles are shown in Figure 2; it depicts that the estimation of the degradation process improved as you have information about more cycles. Even more, this fact is validated in Figure 3, where the mean square error (MSE) of the estimation is calculated considering information of different number of cycles. We can see that the MSE quickly decreases as a function of the number of cycles.

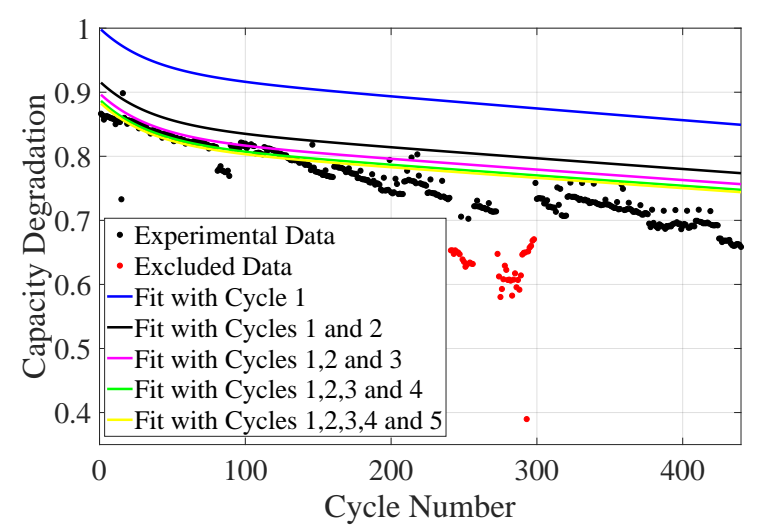

Figure 2. Fitted curves using the estimate of parameters $C$ for different cycles.

\subsection{Estimating the Parameters $c s$ for a Degradation Pro- cess with Different C-Rates}

In this case, it is assumed that the battery is cycled with different C-Rates; consequently, we have to estimate $C_{i}$ for different degradation processes $i=1, \ldots, n$. For instance, if the battery is cycled at $1 \mathrm{C}, 2 \mathrm{C}$ and $3 \mathrm{C}$, we have to estimate $C_{1}, C_{2}$ and $C_{3}$. The main assumption is that each cycle is performed at a fixed discharge current, but this current can be distinct for different cycles. The proposed estimation scheme for this framework is presented in Algorithm 3. It works using the previously introduced Algorithm 2. The idea is to execute 2 in parallel for $n$ different degradation models, where $n$ depends on the number of different discharge currents.

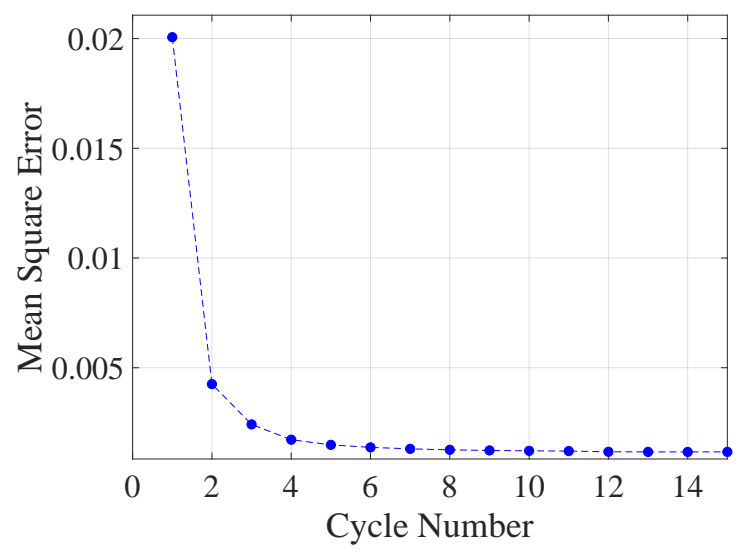

Figure 3. Variation of Mean Square Error as function of the number of cycles.

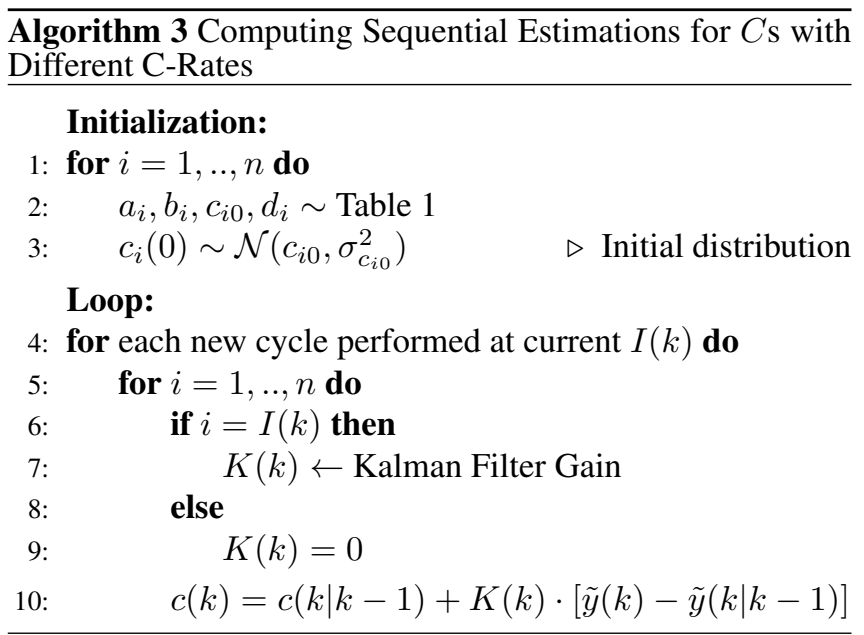

Algorithm 3 is initialized identically than Algorithm 2, that is parameters $a, b, c_{0}$ and $d$ are fixed according to Table 1 (Line 2 ) and a initial normal distribution for $c_{i}(0)$ is assumed (Line $3)$. Then, the loop starts (Line 4), where for each new cycle performed at certain current $I(k)$, is possible to calculate $\tilde{y}(k)$ and to apply KF in order to compute the estimated state $c_{i}(k)$ (Line 10). This procedure is executed for each discharge current model $(i=1, \ldots, n)$. Nevertheless, in this algorithm the Kalman gain is fixed equal to zero when the current C-Rate is not equal to the corresponding model (Line $9)$. In other words, the algorithm only correct $c_{i}(k)$ when the cycled has been performed at current $i$.

\subsubsection{Example Estimation with Different Discharge Cur- rents}

A degradation process at different discharge currents is simulated in order to test the estimation capability of Algorithm 3. This simulation model was built as follows. Firstly, a Markov 
Chain was created so as to generate a series of discharge currents that could be $1 \mathrm{C}, 2 \mathrm{C}$ or $3 \mathrm{C}$, see Figure 4 .

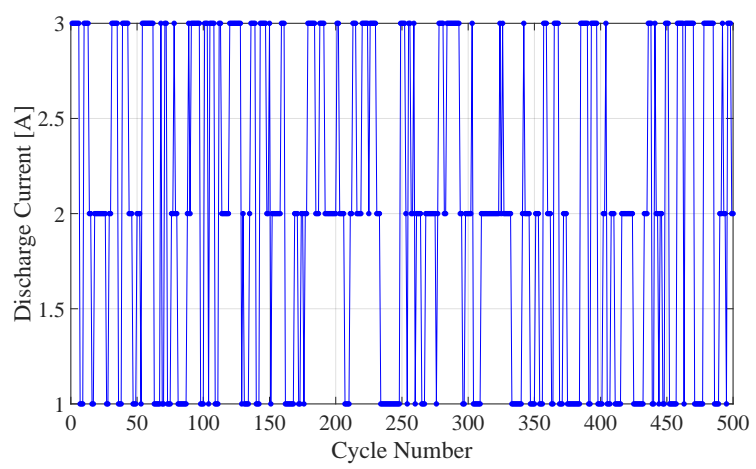

Figure 4. Discharge Currents

Secondly, for each discharge current there was one known degradation model defined according to Eq. (14). Therefore, this model can be employed as ground truth.

$$
y_{i}(k)=a_{i} \cdot e^{b_{i} k}+c_{i} \cdot e^{d_{i} k} i=1,2,3
$$

The simulated degradation process is depicted in Figure 5. We assume that it is not possible to measure the real delivered capacity due to noise measurements. Thus, instead of the real delivered capacity, we measure a delivered capacity corrupted by noise. This fact is illustrated in Figure 5, where the blue line is the truth value and the red line is the measured value.

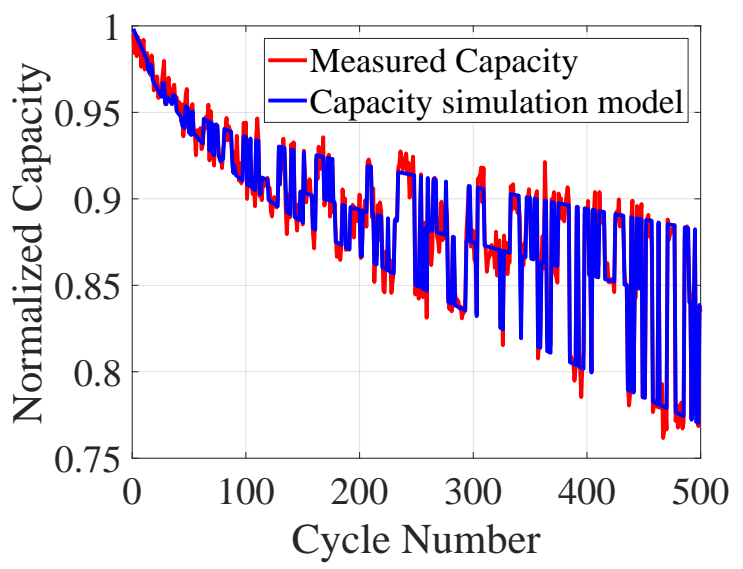

Figure 5. Delivered capacity

Having simulated the complete degradation process, Algorithm 3 is tested with this synthetic data. The results of estimating $c_{1}, c_{2}$ and $c_{3}$ are shown in Figure 6. We can see that even though our estimation algorithm starts with wrong initial conditions, these parameters are learned as soon as new cycles are available. Even more, at cycle 80, the algorithm has already converged to the real values.
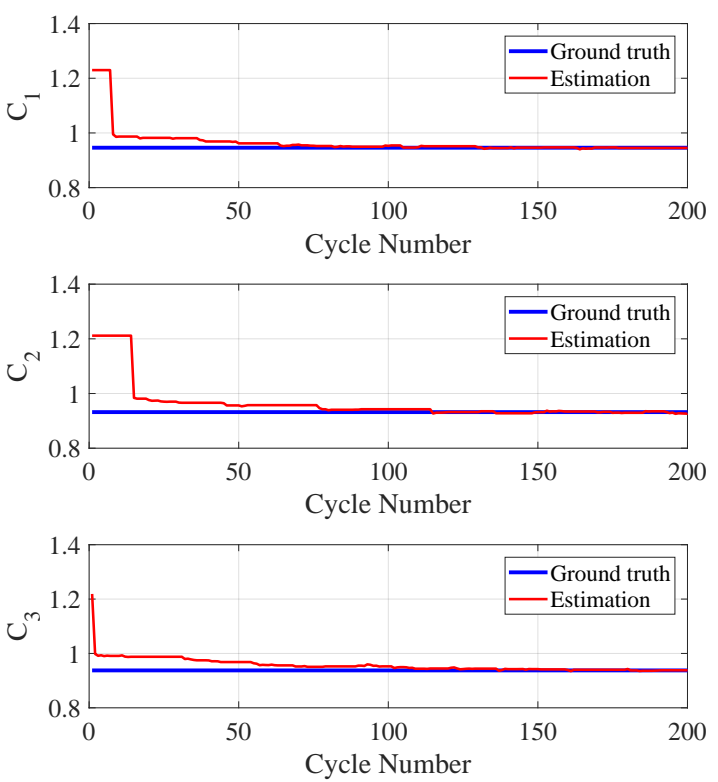

Figure 6. Results of sequential estimation of $c$ s for each degradation model.

\section{Case Study}

\subsection{Generating a Battery Degradation Model from a Datasheet}

As mentioned previously, the degradation process of batteries is highly complex to model due to the huge number of operating variables involved in the process; as a consequence proposing a simulation engine to recreate a degradation process is not a straightforward task. In this section a model to simulate a degradation process is presented.

Overall, battery manufacturers only provide the information of the degradation processes for a determined and fixed CRate. Nevertheless, in practical applications, batteries are discharged at different currents. Taking into account the above, in this section we will introduce a battery degradation process simulation model that considers different discharge currents. This model is based on the information provided by the battery's manufacturer. It works as follows.

1. The degradation curve provided by the manufacturer is used to fit the degradation model presented in Eq. (6)(8). From this, we obtain $a, b, c$, and $d$ as output of this step.

2. Given that only one degradation curve is provided by the manufacturer, the degradation curves at different discharge currents are obtained by scaling the parameters 
obtained in the previous step $(a, b, c$ and $d)$ with the parameters of Table 1.

For a better understanding of the proposed procedure, the next lines illustrate an example. First, we select a battery whose manufacturer provides the degradation curve at $2 \mathrm{C}$, as shown in Figure 7. Then, we fit the degradation model presented in Eq. (6)-(8) to calculate the corresponding parameters $a_{2 C}, b_{2 C}, c_{2 C}$ and $d_{2 C}$. The obtained model after the fitting process is also depicted in Figure 7.

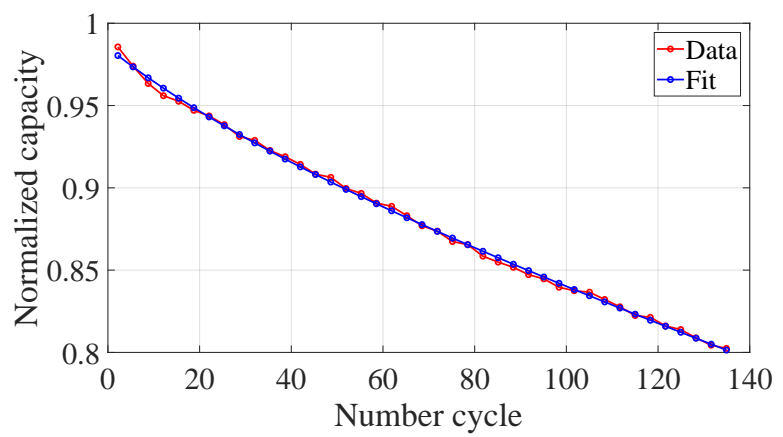

Figure 7. Fitting the degradation model to the degradation information provided by the manufacturer

Afterwards, we calculate the parameters of the degradation model for $1 C$ and $3 C$ using as a reference a scaling-based method using the values of Table 1 . For instance, in the case of $1 C$ :

$$
\frac{a_{1 C}}{a_{1 C}^{T a b l e}}=\frac{a_{2 C}}{a_{2 C}^{T a b l e}}
$$

where $a_{n C}^{T a b l e}$ is the parameter $a$ at $n C$ of Table 1 . Note that $a_{2 C}$ is known.This procedure is repeated for the other parameters at different currents. The underlying assumption of this scaling method is that the degradation parameters of the battery under study are related by a direct proportion with the degradation parameters of the battery of Table 1 . Finally, the resulting degradation processes for different discharge currents for the battery under study are shown in Figure 8.

\subsection{Application of the Proposed Model to an EV Driving Profile}

To understand how the degradation process of a Li-ion battery varies when diverse operation conditions are present, the information obtained from a simulated driving profile of an EV is used. From this profile, information such as the discharge currents (in terms of C-Rate), along with the corresponding SOC values, an SBM framework approach is used. The basis of this approach is presented in (Perez, Jaramillo, et al., 2018). For this approach the three curves of the different discharge processes are required (in case an user has more information, the additional information can be included). Once the

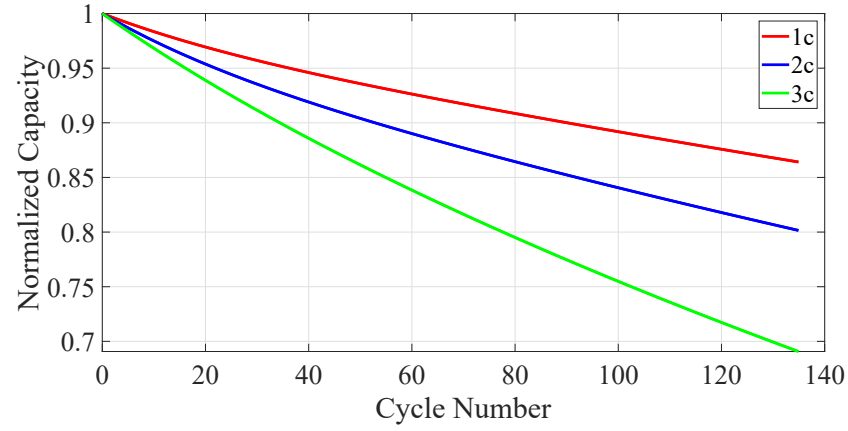

Figure 8. Results of sequential estimation of $c$ s for each degradation model.

three curves obtained in Figure 8 are available, the next step is to calculate the Coulombic efficiency for each case. Figure 9 illustrates the equivalent Coulombic efficiency for each Crate. These values are going to be used as the $D_{o}$ vector of the SBM methodology.

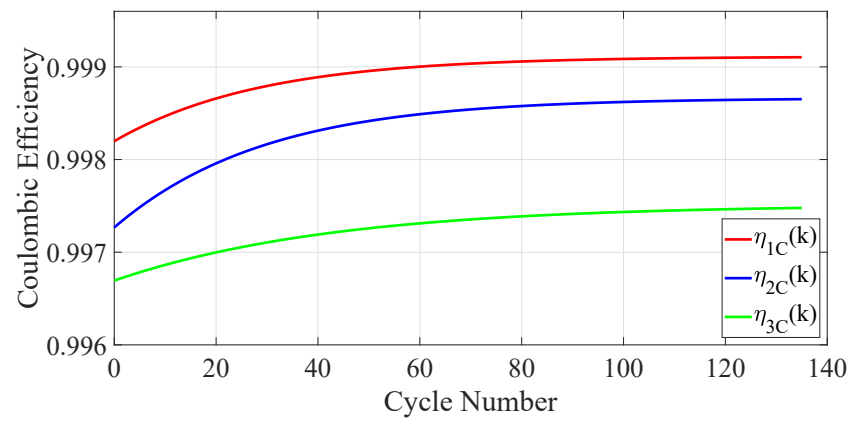

Figure 9. Evolution of the Coulombic efficiency for different C-rates.

In this approach, SBM extrapolates an equivalent degradation factor using the available information when similar conditions are present. Figure 10 shows the delivered normalized capacity for three realizations when random C-Rates, with values between 1 and 3, and variable SOC values are present in each cycle.

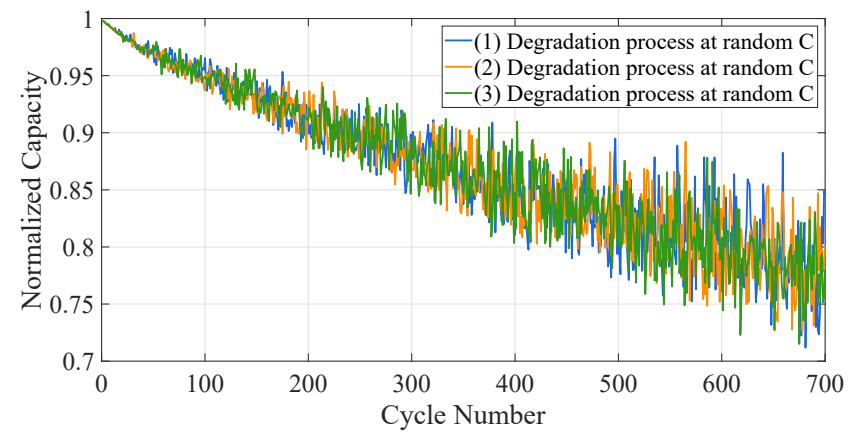

Figure 10. Evolution of the delivered capacity for three realizations. 
From Figure 10 it can be noted how the delivered capacity of each realization is bounded between two dominant trends. This becomes of great importance for instance when an user wants to change the application of the battery to other types of application where less current is required for example, namely second life application for batteries.

To understand the result of the simulation in a clearer manner, Figure 11 illustrates one realization and less cycles. In this figure it can be easily appreciated the degradation process trends and the normalized value of the delivered capacities for each of the cycles. As expected, for the initial cycles there is almost no variance of the delivered values, but as the battery becomes more degraded the variation is more notorious.

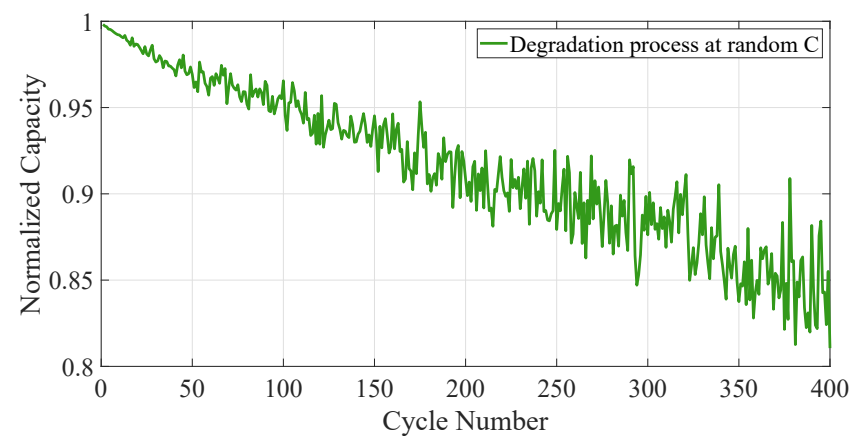

Figure 11. Evolution of the delivered capacity for one realization.

It is important to always keep in mind that the degradation process of a Li-ion is only one, although sometimes it can be confused with the delivered capacity due to their similarity. For example, in a given cycle a battery is discharged at a constant $2 \mathrm{C}$ rate, it is possible that the delivered capacity is less than at $1 \mathrm{C}$ conditions, due to the internal chemical reactions that cause the voltage to cut-off the discharge, or even due to the increase of the internal temperature of the battery. But in the immediate next cycle, if the discharge conditions are modified to a fraction of the current of the previous cycle it would appear that the battery regenerated, however that is a completely different phenomena.

\section{Conclusions}

In this article, a simulation engine that characterizes the degradation process of $\mathrm{Li}$-ion batteries was proposed. The previously proposed model combined with KF can use the information provided by the manufacturer's datasheet regarding the degradation process at nominal and controlled conditions, with the purpose of creating expected degradation conditions based on the performance of other $\mathrm{Li}$-ion batteries. In particular, this model learns its parameters as new cycles are performed using KF to accomplish this goal. Furthermore, an SBM scheme was included to perform the analysis of heterogeneous operating conditions, specifically C-Rates and vari- ant SOC. This type of analysis enables the creation of a simulation engine for the degradation process or deliverable capacity of Li-ion battery. Consequently, one application of this simulation engine is to assist the evaluation of the lifetime of batteries under diverse operating conditions. Moreover, this simulation engine can assist the decision making process for second life applications of batteries. The application of the simulation engine was illustrated with the driving profile of an EV. These results illustrate the delivered capacity along the degradation trend. Moreover, given a profile of future operating loads, this model might be used for EoL prognostic purposes; nevertheless, this idea will be studied in future work.

\section{ACKNOWLEDGMENT}

This work has been partially supported by FONDECYT Chile Grant Nr. 1170044, Conicyt PIA ACT1405 and the Advanced Center for Electrical and Electronic Engineering, AC3E, Basal Project FB0008, CONICYT. The work of Aramis Prez was supported by the University of Costa Rica (Grant for Doctoral Studies) and CONICYT-PCHA/Doctorado Nacional/2015-21150121. The work of Heraldo Rozas was supported by CONICYT-PFCHA/MagisterNacional/201822180232. The work of Francisco Jaramillo was supported by CONICYT-PCHA/Doctorado Nacional/2014-21140201. The work of Vanessa Quintero was supported by the Universidad Tecnológica de Panamá and IFARHU (Grant for Doctoral Studies), CONICYT-PCHA/Doctorado Nacional/201621161427 and SNI-SENACYT.

\section{REFERENCES}

Arulampalam, M. S., Maskell, S., Gordon, N., \& Clapp, T. (2002). A tutorial on particle filters for online nonlinear/non-Gaussian Bayesian tracking. IEEE Transactions on Signal Processing, 50(2), 174-188. doi: 10.1109/78.978374

Bishop, G., Welch, G., et al. (2001). An introduction to the kalman filter. Proc of SIGGRAPH, Course, 8(275993175), 59.

Candy, J. V. (2016). Bayesian Signal Processing: Classical, Modern, and Particle Filtering Methods. Wiley. doi: 10.1002/9781119125495

Charkhgard, M., \& Farrokhi, M. (2010). State-of-charge estimation for lithium-ion batteries using neural networks and ekf. IEEE transactions on industrial electronics, 57(12), 4178-4187.

Fotouhi, A., Auger, D. J., Propp, K., Longo, S., \& Wild, M. (2016). A review on electric vehicle battery modelling: From lithium-ion toward lithium-sulphur. Renewable and Sustainable Energy Reviews, 56, 1008-1021.

Guha, A., \& Patra, A. (2018). State of health estimation of lithium-ion batteries using capacity fade and inter- 
nal resistance growth models. IEEE Transactions on Transportation Electrification, 4(1), 135-146.

Kalman, R. E. (1960). A new approach to linear filtering and prediction problems. Journal of Fluids Engineering, 82(1), 35-45.

Marins, M. A., Ribeiro, F. M., Netto, S. L., \& da Silva, E. A. (2018). Improved similarity-based modeling for the classification of rotating-machine failures. Journal of the Franklin Institute, 355(4), 1913-1930.

Ning, G., \& Popov, B. N. (2004). Cycle life modeling of lithium-ion batteries. Journal of The Electrochemical Society, 151(10), A1584-A1591.

Olivares, B. E., Munoz, M. A. C., Orchard, M. E., \& Silva, J. F. (2013). Particle-filtering-based prognosis framework for energy storage devices with a statistical characterization of state-of-health regeneration phenomena. IEEE Transactions on Instrumentation and Measurement, 62(2), 364-376.

Perez, A., Jaramillo, F., Quintero, V., \& Orchard, M. (2018). Characterizing the degradation process of lithium-ion batteries using a similarity-based-modeling approach. In Phm society european conference (Vol. 4).

Perez, A., Quintero, V., Jaramillo, F., Rozas, H., Jimenez, D., Orchard, M., \& Moreno, R. (2018). Characterization of the degradation process of lithium-ion batteries when discharged at different current rates. Proceedings of the Institution of Mechanical Engineers, Part I: Journal of Systems and Control Engineering, 232(8), 1075-1089.

Pérez, A., Quintero, V., Rozas, H., Jaramillo, F., Moreno, R., \& Orchard, M. (2017). Modelling the degradation process of lithium-ion batteries when operating at erratic state-of-charge swing ranges. In Control, decision and information technologies (codit), 2017 4th international conference on (pp. 0860-0865).

Pola, D. A., Navarrete, H. F., Orchard, M. E., Rabié, R. S., Cerda, M. A., Olivares, B. E., ... Pérez, A. (2015). Particle-filtering-based discharge time prognosis for lithium-ion batteries with a statistical characterization of use profiles. IEEE Transactions on Reliability, 64(2), 710-720.

Salkind, A. J., Fennie, C., Singh, P., Atwater, T., \& Reisner, D. E. (1999). Determination of state-of-charge and state-of-health of batteries by fuzzy logic methodology. Journal of Power sources, 80(1-2), 293-300.

Vachtsevanos, G., Lewis, F., Roemer, M., Hess, A., \& Wu, B. (2007). Intelligent Fault Diagnosis and Prognosis for Engineering Systems. Georgia Institute of Technology, United States.

Yang, F., Wang, D., Xing, Y., \& Tsui, K.-L. (2017). Prognos- tics of li(nimnco)o2-based lithium-ion batteries using a novel battery degradation model. Microelectronics Reliability, 70, 70-78.

\section{BIOGRAPHIES}

Dr. Aramis Perez received his B.Sc. degree and Licentiate degree in Electrical Engineering from the University of Costa Rica. He received his master degree in Business Administration with a General Management Major from the same university. He also received the Doctorate in Electrical Engineering degree from the University of Chile. Currently he is a Research Fellow at the Fault Diagnosis and Failure Prognosis Laboratory and the Energy Center of the University of Chile. Also,he is a Professor at the School of Electrical Engineering at the University of Costa Rica. His research interests include parametric/non-parametric modeling, system identification, data analysis, and manufacturing processes.

M. Sc. Heraldo Rozas received the B.Sc and M.Sc. degree in Electrical Engineering at the University of Chile, in 2017 and 2019, respectively. Currently he is a Research Assistant at the Fault Diagnosis and Failure Prognosis Laboratory of the University of Chile. His research interests include Decision-Making under uncertainty, Diagnostic and Prognostic of faults.

Dr. Francisco Jaramillo received his B.Sc. degree in Electronics Engineering from Universidad de La Frontera, Temuco, Chile. He also received the Doctorate in Electrical Engineering degree from the University of Chile. Currently he is a researcher at the Fault Diagnosis and Failure Prognosis Laboratory at the University of Chile. His research interests include machine learning, control systems, and fault diagnosis and failure prognosis based on Bayesian algorithms with applications to nitrogen removal in pilot-scale Sequencing Batch Reactors for Wastewater Treatment Plants, and wind turbine blades.

Dr. Vanessa Quintero received her B.Sc degree in Electronics and Telecommunication Engineering at the Universidad Tecnológica de Panamá (2007). She received her Doctorate in Electrical Engineering degree from the University of Chile. Currently she is a Professor at Electrical Engineering Faculty at Universidad Tecnológica de Panamá. Her research interests include estimation, prognostics with applications to battery and protocols design.

Dr. Marcos E. Orchard received the B.S. degree and a Civil Industrial Engineering degree with electrical major from Catholic University of Chile. He also received the M.S. and $\mathrm{Ph} . \mathrm{D}$. degrees from The Georgia Institute of Technology, Atlanta, GA, USA. He is currently an Associate Professor with the Department of Electrical Engineering, and the head of the Fault Diagnosis and Failure Prognosis Laboratory at the University of Chile. His current research interest is the design, implementation and testing of real-time frameworks for fault diagnosis and failure prognosis, with applications to battery management systems, mining industry, and finance. 